\title{
Vertebral Artery
}

National Cancer Institute

\section{Source}

National Cancer Institute. Vertebral Artery. NCI Thesaurus. Code C12819.

The first branch of the subclavian artery that ascends both sides of the neck and merges at the middle line to from the basilar artery at the level of the pons. 